\title{
NEW MICROBIOME-BALANCING TOOTHPASTE LAUNCHED IN THE UK
}

Zendium is a toothpaste that works differently. In addition to fluoride, its unique SLS-free formulation contains natural enzymes and proteins which boost the natural salivary defences ${ }^{1}$ and balance the oral microbiome. A landmark study, published in 2017, showed that over 14 weeks' use, Zendium significantly increased health-associated bacteria and significantly reduced disease-associated bacteria. ${ }^{2}$

These findings complement a growing expert consensus which recognises that it is the overall balance of the oral microbiome which is key to oral health rather than the simple presence or absence of a specific pathogen. ${ }^{3}$

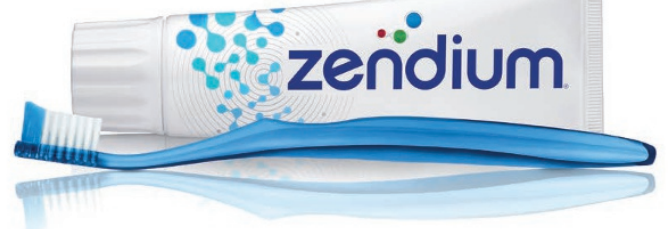

Two further studies now demonstrate the clinical benefit of Zendium's balancing effect. In a 13-week controlled clinical trial, $83 \%$ of Zendium users experienced improved gingival health with Zendium showing superiority vs control across all three clinical indices measured. ${ }^{4}$ Supporting these results, a real-world study of over 300 people in Denmark revealed that long-term Zendium users had significantly better gingival health than those using regular toothpastes. ${ }^{5}$

Zendium can be bought on Amazon.

1. Unilever data on file

2. Adams S E, Arnold D, Murphy B et al. A randomised clinical study to determine the effect of a toothpaste containing enzymes and proteins on plaque oral microbiome ecology. Sci Rep 2017; 7: 43344.

3. Kilian M, Chapple I L, Hannig M et al. The oral microbiome - an update for oral healthcare professionals. Br Dent J 2016; 221: 657-666.

4. West N, Newcombe R, Mantzourani M et al. A toothpaste containing enzymes and proteins improves gingival health. In CED-IADR/NOF Oral Health Research Congress September 21-13, 2017: Abstract Book. 0527. International Association for Dental Research, 2017.

5. Pedersen A L, Belstrøm D, Nicholson J et al. Gingival health status in individuals using different types of toothpaste. In CED-IADR/NOF Oral Health Research Congress September 21-13, 2017: Abstract Book. 0472. International Association for Dental Research, 2017.

\section{ZENDIUM COMPLETE PROTECTION REVIEW}

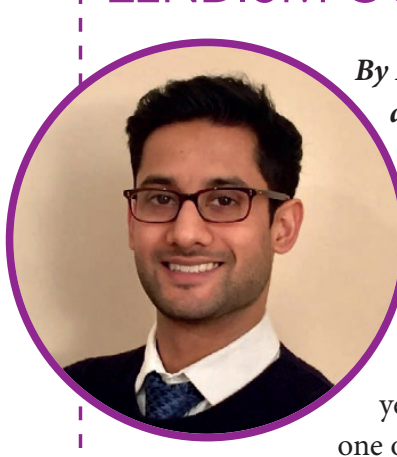

By Kishan Patel, dental therapist Zendium is a toothpaste brand that has been developed in Scandinavia over the past 40 years where it is also one of the toothpastes most recommended by dental professionals ${ }^{1}$. Zendium contains active enzymes and proteins that are found in saliva, with the aim of boosting the body's natural defences to keep the mouth's microflora balanced. This results in a reduction in the number of diseaseassociated bacteria and an increase in the number of health-associated bacteria ${ }^{2}$, therefore antibacterial agents such as triclosan are not required to reduce bacterial numbers. Fluoride is present at $1450 \mathrm{ppm}$ in the form of sodium fluoride, aiding remineralisation of enamel and providing protection against cavities ${ }^{3}$.

All Zendium toothpastes are free of sodium lauryl sulphate (SLS). SLS is a common foaming agent which has been known to cause aphthous stomatitis and irritations to mucosa in the mouth ${ }^{4}$. SLS is also known to alter taste for a short period after use and may cause xerostomia ${ }^{5}$.
As Zendium is SLS free it can also be used together with products containing chlorhexidine digluconate if required. SLS reduces the antiplaque effect of products containing chlohexidine digluconate ${ }^{6}$. Zendium products are also paraben free. Zendium Complete Protection has a mild, subtle peppermint taste with a minimal cooling effect, not leaving the mouth overwhelmed with a minty taste, as found with many other toothpastes. It lightly foams in the mouth but significantly less than toothpastes which contain SLS, and is also not abrasive in texture. I appreciate its method of delivering protection for a healthier mouth through the addition of active enzymes and proteins. Zendium is limiting chemicals (SLS, parabens and triclosan) which have been known to be harmful and is keeping mild ingredients in its formulation. This would appeal to those who know these ingredients may be detrimental to their health.

Zendium Complete Protection would appeal to:

- Those who suffer from aphthous stomatitis, irritations of the mucosa or xerostomia linked to SLS

- Those who suffer from sensitive gingiva and/or suffer from gingivitis or periodontitis
- Those who prefer a mild tasting toothpaste

- Those who use a chlorhexidine digluconate product during their oral hygiene routine

- Those looking for a paraben free and/or SLS free and/or triclosan free toothpaste which contains fluoride

1. Zendiumarabia.com. (2018). Welcome to Zendium Arabia. [online] Available at: https://www.zendiumarabia.com/ en [Accessed 16 Sep. 2018].

2. Adams, S., Arnold, D., Murphy, B., Carroll, P., Green, A., Smith, A., Marsh, P., Chen, T., Marriott, R. and Brading, M. (2017). A randomised clinical study to determine the effect of a toothpaste containing enzymes and proteins on plaque oral microbiome ecology.

3. Banerjee, A., Watson, T., Kidd, E. and Pickard, H. (2011). Pickard's manual of operative dentistry. Oxford: Oxford University Press.

4. Natalie Rose Edgar, R. (2017). Recurrent Aphthous Stomatitis: A Review. [online] PubMed Central (PMC). Available at: https://www.ncbi.nlm.nih.gov/pmc/ articles/PMC5367879/ [Accessed 16 Sep. 2018].

5. Haveman C, Xerostomia Management in the Head and Neck Radiation Patient. Texas Dental Journal, June 2004 484-497.

6. Barkvoll, P., Rolla, G. and Svendsen, K. (1989). Interaction between chlorhexidine digluconate and sodium lauryl sulfate in vivo. - PubMed - NCBI. [online] Ncbi.nIm.nih. gov. Available at: https://www.ncbi.nlm.nih. gov/pubmed/2794095 [Accessed 16 Sep. 2018]. 